(c) 2017 IEEE, P. Wang, C. Huang, J. C. Tilton, B. Tan and E. C. B. de Colstoun, "HOTEX: An approach for global mapping of human built-up and settlement extent," 2017 IEEE International Geoscience and Remote Sensing Symposium (IGARSS), Fort Worth, TX, 2017, pp. 1562-1565. doi: 10.1109/IGARSS.2017.8127268.

\title{
HOTEX: AN APPROACH FOR GLOBAL MAPPING OF HUMAN BUILT-UP AND SETTLEMENT EXTENT
}

\author{
Panshi Wang ${ }^{a}$, Chengquan Huang ${ }^{a}$, James C. Tilton ${ }^{b}$, Bin Tan ${ }^{c}$, Eric C. Brown de Colstoun ${ }^{d}$ \\ a Department of Geographical Sciences, University of Maryland, College Park, MD, USA \\ *pswang@umd.edu \\ b Computational and Information Sciences and Technology Office, NASA GSFC, Greenbelt, MD 20771 USA \\ c Science Systems and Applications, Inc., NASA GSFC, Greenbelt, MD 20771 USA \\ d Biospheric Sciences Laboratory, NASA GSFC, Greenbelt, MD 20771 USA
}

\begin{abstract}
Understanding the impacts of urbanization requires accurate and updatable urban extent maps. Here we present an algorithm for mapping urban extent at global scale using Landsat data. An innovative hierarchical object-based texture (HOTex) classification approach was designed to overcome spectral confusion between urban and nonurban land cover types. VIIRS nightlights data and MODIS vegetation index datasets are integrated as high-level features under an object-based framework. We applied the HOTex method to the GLS-2010 Landsat images to produce a global map of human built-up and settlement extent. As shown by visual assessments, our method could effectively map urban extent and generate consistent results using images with inconsistent acquisition time and vegetation phenology. Using scene-level cross validation for results in Europe, we assessed the performance of HOTex and achieved a kappa coefficient of 0.91 , compared to 0.74 from a baseline method using per-pixel classification using spectral information.
\end{abstract}

Index Terms - Object-based Classification, Hieratical Segmentation, Texture, Urban, Landsat

\section{INTRODUCTION}

Urbanization has broad impacts on the Earth's environmental systems [1]. Understanding the drivers, impacts and feedbacks of urban growth requires detailed and up-to-date information on the spatial extent of urban areas. Coarse to medium resolution remote sensing datasets, such as the Defense Meteorological Satellite Program Operational Linescan System (DMSP-OLS) and Moderate Resolution Imaging Spectroradiometer (MODIS)), have been used to map urban extent at global scale [2, 3]. However, the complexity of urban landscape requires much finer resolution data sources to be accurately mapped [4]. Publicly available Landsat-grade datasets are the optimal sources for mapping urban extent at global scale. Global orthorectified cloud-free
Landsat datasets have been collected through Global Landsat Survey (GLS) [5], which has greatly reduced the difficulty of global urban mapping.

Most pixels within the urban extent are mixtures of urban and non-urban surfaces. Even for pixels with a single type of surface cover, they often have similar spectral signatures as the same cover type in non-urban areas. Another challenge to Landsat-based urban mapping is the inconsistency of vegetation phenology among the images available. In this case, deciduous vegetation and non-vegetated surfaces may be difficult to separate. Further, classification of adjacent images with different vegetation phenology using spectralbased methods can result in artifacts and discontinuities along image boundaries.

The main purpose of this study is to develop an approach suitable for global scale mapping of human builtup and settlement extent (HBASE) using the GLS images. The HBASE definition includes all types of human built-up surfaces and the areas surrounding them that are functionality linked to those surfaces (e.g., urban green spaces). Building on past efforts to meet the challenges of urban mapping using spatial image features such as edge density and gray level co-occurrence matrix (GLCM) [6], we introduce here a method based on hierarchical object-based textures (HOTex) using texture measures derived from objects at multiple levels of segmentation hierarchy and integrating high-level features based on the Visible Infrared Imaging Radiometer Suite (VIIRS) Day/Night Band (DNB) and MODIS vegetation index(VI) datasets.

\section{METHODOLOGY}

The HOTex method consists of the following major components: derivation of hierarchical image segments, texture calculation, high-level feature extraction, training and classification, and post-classification processing (Fig. 1). In this study, the GLS-2010 images were used to derive image segments and texture measures. Nightlight data obtained from the VIIRS DNB band and a MODIS VI data set were 
used to derive high level features in order to improve classification performance. The OpenStreetMap road network dataset was used in post-classification processing to improve representation of major roads in the derived HBASE product.

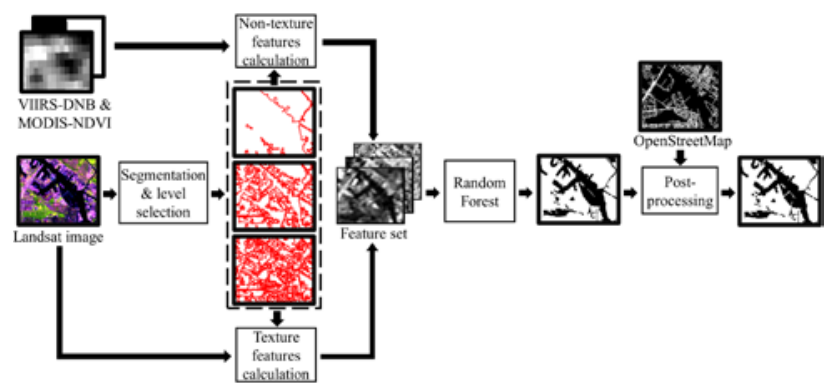

Figure 1. Overall workflow of the HOTex method.

Image segmentation was performed using the Recursive Hierarchical Image Segmentation (RHSeg) software package [7], a recursive approximation of the Hierarchical Image Segmentation (HSeg) package. HSeg combines the power of best merge region growing to delineate the boundaries between spatially adjacent region and spectral clustering to group spatially disjoint regions together. RHSeg was designed to improve the speed of the HSeg algorithm on cluster or cloud computing systems, which made it possible processing all Landsat images required by this study. The output of RHSeg algorithm includes image objects at multiple scales, where finer scale objects are nested within coarser scale objects. In this study, we used object size thresholds to select three representative levels from the RHSeg segmentation hierarchy.

At each level, we calculated GLCM features including angular second moment, contrast, correlation, and variance using objects instead of windows as spatial units. In addition to traditional single-band texture features, we also extended the concept of GLCM by using the co-occurrence between two Landsat bands to calculate cross-band (color) texture features. The single-band textures are calculated for the Landsat spectral bands 5, 4 and 3. Since our implementation of GLCM is symmetrical, the cross-band textures are only calculated for the band combinations $(5,4)$, $(4,3)$ and $(5,3)$.

Three groups of variables were derived in addition to the textures. The first was a binary variable indicating whether an image was a Landsat 7 image, in order to separate images with and without SLC-off gaps. We used metrics derived from MODIS normalized difference vegetation index (NDVI) datasets, including annual maximum, annual median, and the NDVI value for the month when the corresponding Landsat image was acquired. And finally we used statistics derived from VIIRS DNB datasets.

We collected training samples of HBASE/non-HBASE image objects by interpreting of Landsat images. Different sets of features were used as inputs to train random forest classification models in order to determine the best feature set for mapping HBASE. For comparison, we also tested a baseline method using spectral features only, which are the Landsat bands, the tasseled cap transform, and NDVI.

\section{RESULTS}

\subsection{Mapping Results and visual assessments}

First, we visually examined the HBASE products derived using the HOTex approach against the results the baseline method using spectral features (Fig. 2). We found that the HOTex method generates consistently better results than the baseline method in terms of the compactness of built-up and settlement extent, the removal of commission errors in areas such as agricultural fields and bare lands, and the inclusion of low density built-up areas and settlements.
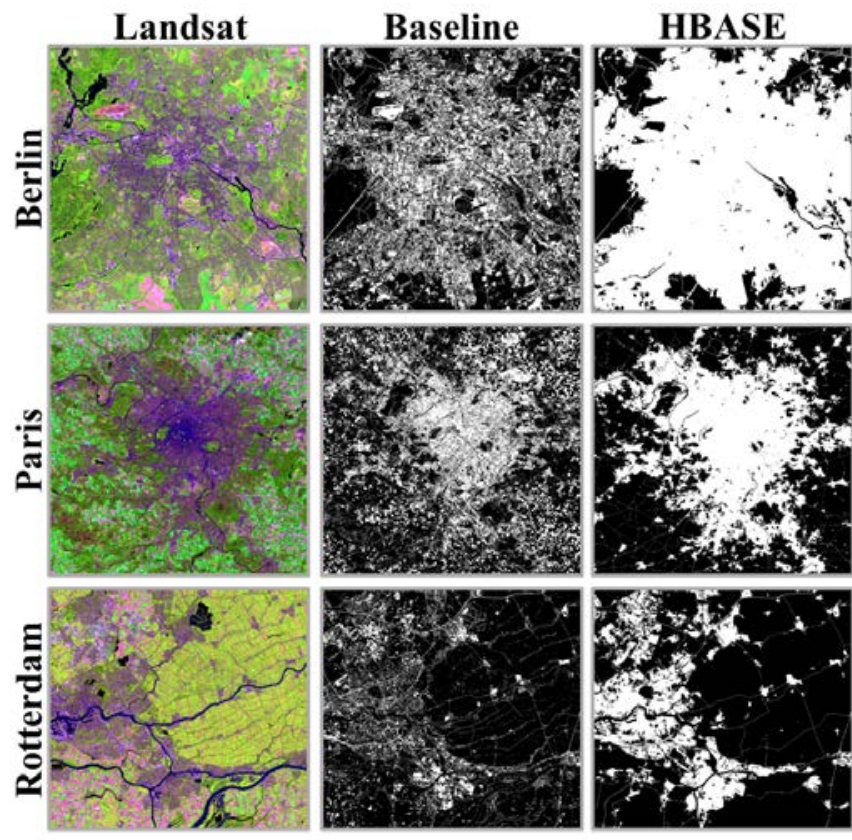

Figure 2. Comparis ons of HBASE classifications derived using the baseline method and the HOTex method.

Then, we examined the overall performance of our method over Europe by creating a continental mosaic of HBASE maps (Fig. 3). As shown by an example of the mapping results for two adjacent Landsat scenes, the spectral-based baseline method is sensitive to seasonal differences in the image and produced a clear difference across the image boundary, while the HOTex method produced consistently accurate results without such artifacts.

Finally, we applied the HOTex method to the entire GLS2010 archive to create global HBASE products. These products are yet to be assessed. However, the overall spatial pattern suggests a robust performance globally (Fig. 4). 


\subsection{Scene-level cross validation}

We performed scene-level cross validation by dividing the Landsat scenes where training data were collected into 10 random groups. Training samples from 9 of the 10 scene groups were used together to train the random forest algorithm, which was then evaluated using reference samples in the set-aside image group. This was repeated 10 times such that each time samples from a different image group were used to evaluate the random forest model derived using samples from the other 9 image groups. We calculated cross validation accuracy scores including overall accuracy, kappa coefficient, as well as producer's and user's accuracies for the HBASE class (Table I).

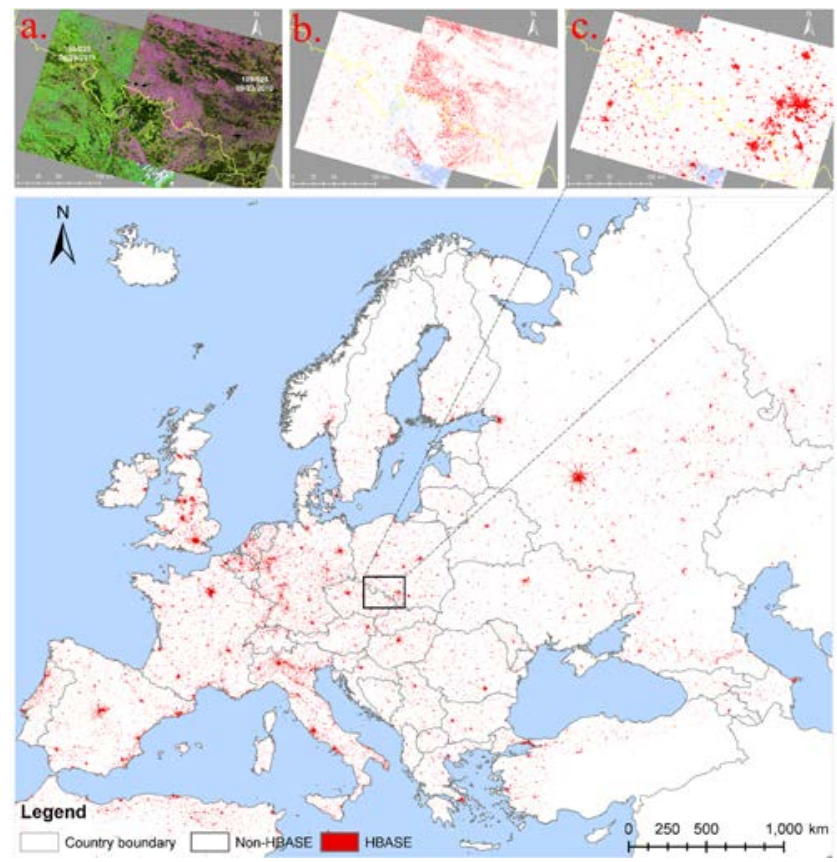

Figure 3. A continental mosaic of HBASE products over Europe and a mosaic for two overlapping Landsat tiles: (a) Landsat images p190r025 and p189r025; (b) spectral-based classification; (c) classification using the HOTex method.

\section{TABLE I}

Scene-level cross validation scores of HBASE classifications using different input feature sets (OA: overall accuracy, UA: user's accuracy, PA: producer's accuracy).

\begin{tabular}{c|cccc}
\multicolumn{1}{c}{ OA } & UA & PA & KAPPA \\
\hline SPECTRAL & $94.0 \%$ & $76.5 \%$ & $78.1 \%$ & 0.74 \\
GLCM & $96.8 \%$ & $86.2 \%$ & $89.9 \%$ & 0.83 \\
GLCM+VI & $97.1 \%$ & $91.1 \%$ & $86.3 \%$ & 0.87 \\
GLCM+DNB & $97.1 \%$ & $86.6 \%$ & $92.3 \%$ & 0.88 \\
GLCM+DNB+VI & $\mathbf{9 7 . 9 \%}$ & $\mathbf{9 1 . 4 \%}$ & $\mathbf{9 2 . 8} \%$ & $\mathbf{0 . 9 1}$ \\
\hline
\end{tabular}

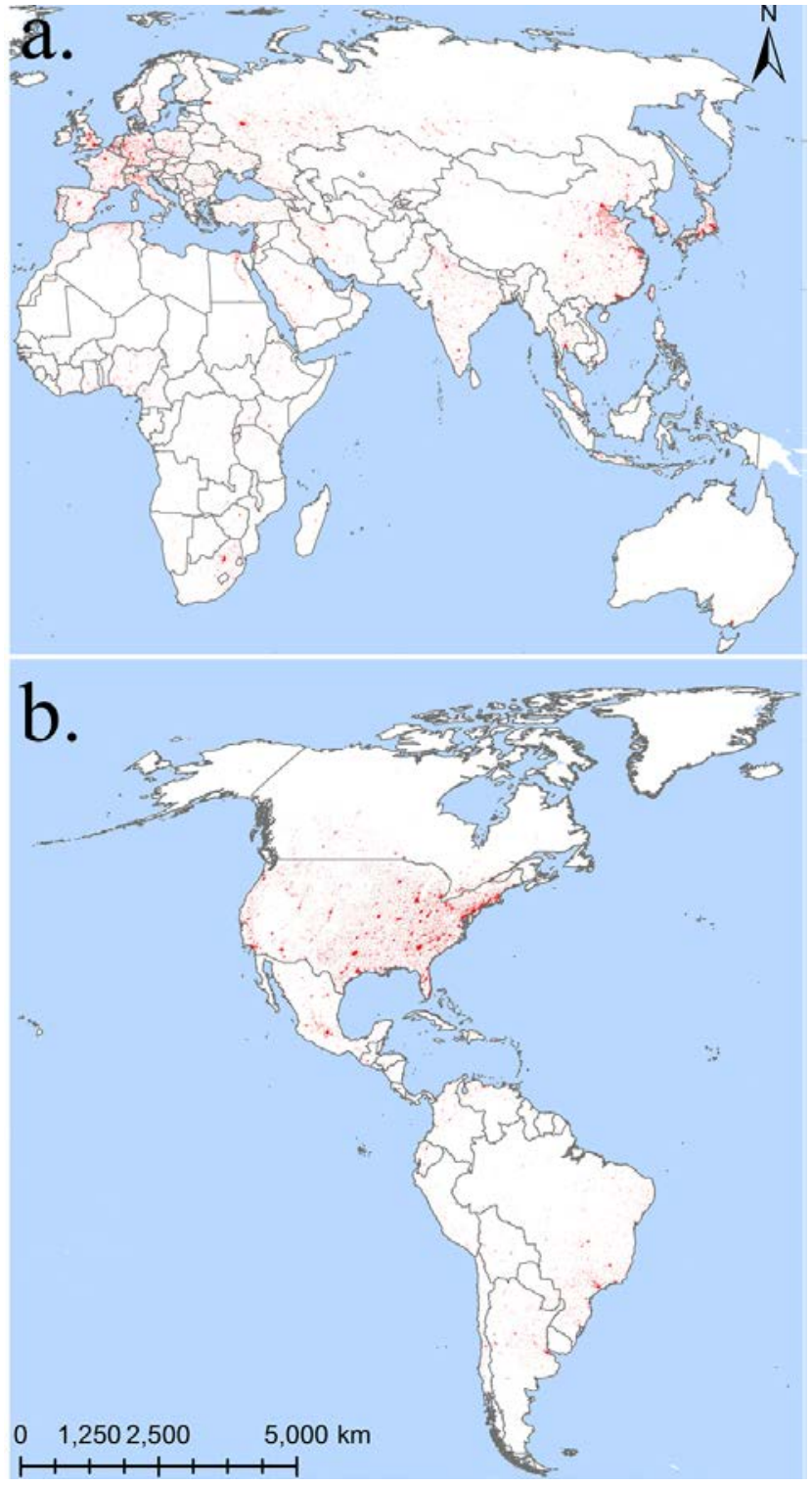

Figure 4. Global 500m mosaic (Robinson projection) of HBASE products produced using the HOTex method and the Global Land Survey 2010 archive: (a) Eurasia, Africa and Australia; (b) North America and South America.

\subsection{Comparison with other urban maps}

We also compared our HBASE maps and other urban products including the $1 \mathrm{~km}$ Global Rural-Urban Mapping Project (GRUMP) dataset [8], the 500m MODIS Collection 5.1 land cover product (MCD12Q1) [3], the 300m GlobCover product [9], and the GlobeLand30 product [10]. The comparisons were done based on the functional urban areas (FUAs) as defined by Urban Atlas 2012 (UA), an urban map for EU-27 countries developed using high-resolution imagery and auxiliary datasets [11]. FUA incorporates cities 
with spatial proximity and functional connection and also open spaces in between. For all the 437 FUAs available at the time of this study, we calculated the size of urban extent within each FUA. The scatterplots in Fig. 5 are urban extent sizes of HBASE (x-axis) plotted against urban extent sizes of the five urban products compared (y-axis). The result shows that MODIS and Globcover products greatly underestimate urban extent compared to UA, while the GRUMP product tend to overestimate. According to the root mean square difference (RMSD) scores, our HBASE product agrees best with the UA product, slightly better than GlobeLand30.

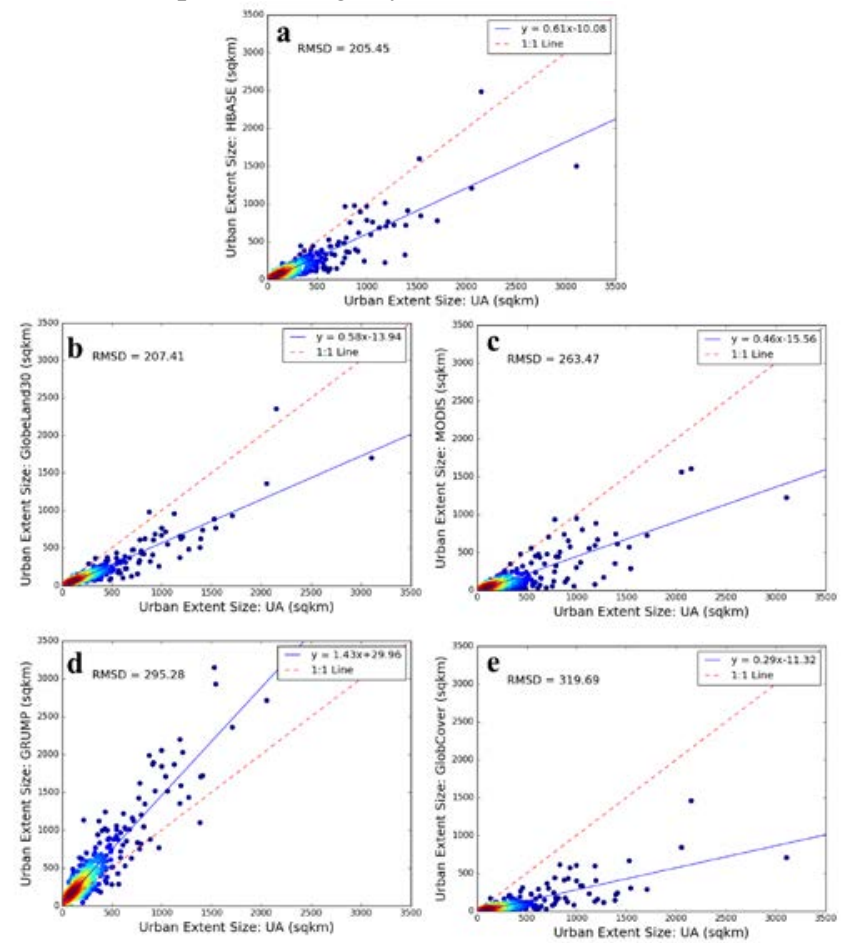

Figure 5. Size of urban atlas (UA) urban extent for the FUAs (x-axis) plotted against size of urban extent from: (a) HBASE, (b) GlobeLand30, (c) MODIS, (d) GRUMP, (e) GlobCover.

\section{CONCLUSION}

A hierarchical object-based texture (HOTex) approach was developed for mapping global human built-up and settlement extent (HBASE). Segmented hierarchical image objects provided a framework for integrating multi-level textures and integrating coarser resolution VIIRS DNB and MODIS VI for classification. According to scene-level cross validation, the kappa, user's and producer's accuracies of HBASE products derived using the proposed algorithm are all over $90 \%$. Visual assessments and comparisons between our HBASE product and other urban products also demonstrated the effectiveness of the HOTex approach for mapping human built-up and settlement areas globally.

\section{ACKNOWLEDGEMENTS}

This study was supported by NASA's Land Cover and Land Use Change (LCLUC) Program (grants 09-LCLUC09-20136-1 \& NNX11AH67G). Additional support for Panshi Wang and Chengquan Huang was provided by NOAA and US Geological Survey.

\section{REFERENCES}

[1] J. A. Foley, R. Defries, G. P. Asner, C. Barford, G. Bonan, S. R. Carpenter, et al., "Global Consequences of Land Use," Science, vol. 309, pp. 570-4, 2005.

[2] M. L. Imhoff, W. T. Lawrence, D. C. Stutzer, and C. D. Elvidge, "A Technique for Using Composite Dmsp/Ols "City Lights" Satellite Data to Map Urban Area," Remote Sensing of Environment, vol. 61, pp. 361-370, 1997.

[3] M. A. Friedl, D. Sulla-Menashe, B. Tan, A. Schneider, N. Ramankutty, A. Sibley, et al., "Modis Collection 5 Global Land Cover: Algorithm Refinements and Characterization of New Datasets," Remote Sensing of Environment, vol. 114, pp. 168-182, 2010.

[4] Q. Weng, "Remote Sensing of Impervious Surfaces in the Urban Areas: Requirements, Methods, and Trends," Remote Sensing of Environment, vol. 117, pp. 34-49, 2012.

[5] G. Gutman, C. Huang, G. Chander, P. Noojipady, and J. G. Masek, "Assessment of the Nasa-Usgs Global Land Survey (Gls) Datasets," Remote Sensing of Environment, vol. 134, pp. 249-265, 2013.

[6] M. Herold, X. H. Liu, and K. C. Clarke, "Spatial Metrics and Image Texture for Mapping Urban Land Use," Photogrammetric Engineering and Remote Sensing, vol. 69, pp. 991-1001, 2003.

[7] J. C. Tilton, Y. Tarabalka, P. M. Montesano, and E. Gofman, "Best Merge Region-Growing Segmentation with Integrated Nonadjacent Region Object Aggregation," Ieee Transactions on Geoscience and Remote Sensing, vol. 50, pp. 4454-4467, 2012.

[8] CIESIN (Center for International Earth Science Information Network). Global Rural-Urban Mapping Project, Version 1 (GRUMPv1): Urban Extents. Available online at: http://sedac.ciesin.columbia.edu/data/ collection/grump-v1. 2011.

[9] O. Arino, D. Gross, F. Ranera, M. Leroy, P. Bicheron, C. Brockman, et al., "Globcover: Esa Service for Global Land Cover from Meris," IGARSS: 2007 IEEE International Geoscience and Remote Sensing Symposium, Vols 1-12, pp. 2412-2415, 2007.

[10] J. Chen, J. Chen, A. Liao, X. Cao, L. Chen, X. Chen, et al., "Global Land Cover Mapping at 30m Resolution: A Pok-Based Operational Approach," ISPRS Journal of Photogrammetry and Remote Sensing, vol. 103, pp. 727, 2015.

[11] European Environment Agency (EEA). Urban Atlas 2012. Available online at: http://land.copernicus.eu /local/urban-atlas/urban-atlas-2012 . 2016. 